\title{
Additional Clinical Value for PET/MRI in Oncology: Moving Beyond Simple Diagnosis
}

\author{
Kenneth A. Miles ${ }^{1,2}$, Stefan A. Voo ${ }^{1}$, and Ashley M. Groves ${ }^{1}$ \\ ${ }^{1}$ Institute of Nuclear Medicine, University College London, London, United Kingdom; and ${ }^{2}$ Department of Diagnostic Imaging, \\ Princess Alexandra Hospital, Brisbane, Australia
}

Initial clinical research comparing the diagnostic performance of PET/MRI and PET/CT has largely shown equivalent diagnostic capabilities for these modalities in oncology. These uncertainties about the magnitude of diagnostic benefit are compounded by the considerable health economic challenges associated with clinical implementation. Therefore, there is a need to identify ways to extend the use of this technology beyond simple diagnosis so that PET/MRI can add sufficient clinical value beyond PET/CT or MRI alone and become a costeffective imaging modality in clinical practice. A major advantage of PET/MRI over other imaging modalities is the ability to generate multiple quantitative images from a single examination. This article describes how a multiparametric PET/MRI approach not only can add clinical value through contributing to precision medicine but also can establish PET/MRI as a potentially cost-effective imaging modality in oncology.

Key Words: molecular imaging; oncology; PET/MRI; oncology; positron emission tomography/magnetic resonance imaging; precision medicine

J Nucl Med 2018; 59:1028-1032

DOI: 10.2967/jnumed.117.203612

\section{O}

nce the introduction of clinical PET/MRI in 2010, much of the research into this modality has focused on diagnostic performance. However, comparative studies to date have largely shown equivalent diagnostic capabilities for these modalities $(1,2)$. Where benefits for PET/MRI have been demonstrated, they are typically small or apply to relatively small populations of patients. Furthermore, the extent to which these benefits might be equally achievable through sequentially performed PET/CT and dedicated MRI examinations is often unclear. There is therefore a need to identify PET/MRI applications that provide additional value beyond modest improvements in diagnostic performance.

Received Nov. 1, 2017; revision accepted Mar. 6, 2018

For correspondence or reprints contact: Kenneth A. Miles, Department of

Radiology, Princess Alexandra Hospital, 199 Ipswich Rd., Woolloongabba,

Queensland 4102, Australia.

E-mail: kenneth.miles@ucl.ac.uk

Published online Mar. 15, 2018.

COPYRIGHT @ 2018 by the Society of Nuclear Medicine and Molecular Imaging.

The translation of stand-alone PET from a research technique to a routine clinical modality was predicated on demonstrating cost-effectiveness, and similar requirements can be anticipated for hybrid PET/MRI. There are considerable health economic challenges that need to be overcome before PET/MRI can be widely adopted into clinical practice. Currently, the cost of a PET/MRI machine is comparable to separate purchases of PET/CT and dedicated MRI systems. Substantial incremental health benefits over PET/CT, or significant reductions in equipment costs, will therefore be required for PET/MRI to achieve cost-effectiveness. The economic challenges are further magnified if acquisition times for either modality during PET/MRI extend beyond the time required to obtain images of diagnostic quality for one of the modalities alone. It is highly unlikely that these economic burdens will be offset by any health benefits achieved through the modest improvements in diagnostic performance shown to date, and extending the use of the technology beyond simple diagnosis will be required if PET/MRI is to become a cost-effective imaging modality.

A major advantage of PET/MRI over other imaging modalities is the ability to generate multiple quantitative images from a single examination. In this article, we review how this multiparametric PET/MRI approach not only can add clinical value through contributing to precision medicine but also can establish PET/MRI as a potentially costeffective imaging modality in oncology.

\section{FUNCTIONAL AND MOLECULAR IMAGING IN THE ERA OF PRECISION CANCER MEDICINE}

Precision medicine aims to identify subgroups of patients with similar disease characteristics so that the safest and most effective or cost-effective treatment can be selected for individual patients. Expanding the role of imaging to include acquisition of data that allow these subgroups to be identified as part of routine diagnosis constitutes a major opportunity for radiology to contribute significantly to this new era (3). Both PET and MRI offer specific advantages for engaging with this opportunity, and the potential benefits of combining PET and MRI assessments for precision medicine have been highlighted by image-based prognostic nomograms proposed for patients with cervical cancer (4). Simultaneous image acquisition using PET/MRI can enhance 
this potential by reducing total imaging time and avoiding complex image coregistration techniques.

A major focus for precision medicine in oncology is the identification of patients most likely to respond to drugs that target specific molecular mechanisms. The ability of PET to quantitatively assess molecular biology in vivo therefore makes this modality a logical candidate technique for guiding precision medicine (5). Molecular imaging has several advantages that suggest a complementary role to tissue-based assays such as genomic analysis. Imaging is noninvasive and can assess tissues that are not amenable to biopsy. Heterogeneity of tumor biology within a single tumor site, between tumor sites, and within individual tumors over time represents a significant constraint on tissue analysis of biopsy material due to possible sampling error (6). Conversely, imaging can assess whole tumors as well as multiple disease sites, with the option to repeat assessments over several time points. By depicting the tumor phenotype, molecular imaging reflects not only the tumor genetic landscape but also the expression of particular gene mutations in the biologic behavior. These downstream processes can potentially affect the efficacy of drugs selected on the basis of genetic assays.

There is a wide range of established and novel PET tracers that reflect key aspects of tumor biology with the potential to contribute to precision medicine. Even tumor uptake of ${ }^{18} \mathrm{~F}$ FDG, the most widely used PET radiotracer for oncologic diagnosis, has been associated with several tumor biologic features of therapeutic significance, including mutations of the Kirsten rat sarcoma viral oncogene homolog and angiogenesis-related genes in colorectal cancer $(7,8)$, kit mutations in gastrointestinal stromal tumors (9), p16 status in head and neck cancer $(10,11)$, and p-glycoprotein-mediated multidrug resistance in non-small cell lung cancer and hepatocellular carcinoma $(12,13)$. However, the fact that ${ }^{18} \mathrm{~F}-\mathrm{FDG}$ uptake can reflect several different biologic features indicates a need to combine measurements of ${ }^{18} \mathrm{~F}-\mathrm{FDG}$ uptake with other parameters to improve specificity.

MRI offers a range of quantitative functional imaging techniques that are complementary to PET yet more extensive than is available with CT. Diffusion-weighted imaging can assess tumor cellularity, chemical shift imaging evaluates fractional water content and hypoxic blood volume, and dynamic contrast-enhanced techniques reflect angiogenesis. Contrast enhancement dynamics and apparent diffusion coefficient from diffusion-weighted sequences have been shown to be associated with specific molecular subtypes in breast cancer (14-16) and to be predictive of treatment response in a range of cancers (17). These techniques can therefore potentially contribute to precision medicine in their own right.

\section{IMPLEMENTING MULTIPARAMETRIC PET/MRI FOR PRECISION MEDICINE}

PET/MRI is particularly well suited to a role within precision medicine for several reasons. First, imaging data that would have been acquired for clinical management using PET/CT or whole-body MRI can be obtained concurrently with a broad range of quantitative imaging techniques. Second, the accurate coregistration of PET and MRI datasets afforded by simultaneous image acquisition for the 2 modalities enables derivation of combined PET/MRI parameters. Third, multiple MR acquisitions can be acquired with no increase in radiation dose.

The multiparametric approach to PET/MRI can be extended further by deriving additional quantitative features that reflect the heterogeneity of the biologic process depicted by each PET/MR image through use of image processing techniques such as texture analysis. Although a similar approach is feasible using PET/CT, the number of quantitative images available for texture analysis is much greater for PET/MRI. The potential value of MRI-based texture analysis has been illustrated recently by studies reporting that imaging results correlate with the p53 status in head and neck cancer and survival outcomes in breast cancer $(18,19)$. Methods for deriving prognostic or predictive imaging signatures from the large sets of parameters produced by applying image processing to multiple image acquisitions (sometimes known as radiomics) are currently poorly developed. However, these datasets are amenable to multiparametric analysis methods such as recursive partitioning and principle component analysis. The feasibility of radiomic analysis as applied to PET/MRI data has recently been confirmed by Antunes et al., who described a 3-parameter signature combining fluorothymidine SUV from PET with texture features from T2-weighted images and apparent diffusion coefficient maps from MRI, to maximize detection of early treatment effects in 2 patients with renal cancer (20).

\section{Multiparametric PET/MRI for Precision Medicine in Place of Diagnostic PET/CT}

When multiparametric PET/MRI for precision medicine is implemented in place of a clinically indicated diagnostic PET/CT, a broad range of MRI parameters reflecting various aspects of tumor biology can be acquired with no additional radiation burden. On the other hand, the parameters that can be derived from the PET component of the examination will be constrained by the radiopharmaceutical required for clinical purposes, typically ${ }^{18}$ F-FDG. However, the longer acquisition time required for multiparametric MRI creates an opportunity for simultaneous acquisition of dynamic PET data from which a range of additional parameters reflecting the temporal changes in radiotracer can be derived. Quantitative parameters from dynamic ${ }^{18}$ F-FDG PET have been shown to predict response to chemotherapy in locally advanced breast cancer and metastatic colorectal cancer $(21,22)$. Preclinical studies indicate that dynamic ${ }^{18} \mathrm{~F}-\mathrm{FDG}$ PET may also distinguish between tumors with and without inflammation (23). If confirmed in clinical studies, this capability would suggest a potential role in predicting response to immune checkpoint inhibitor for which the presence of tumor-associated inflammation is considered to be associated with long-lasting response (24). Using PET/CT, derivation of 
these additional parameters would entail a significant extension of the examination time with a corresponding impact on workflows and cost.

Some non- ${ }^{18} \mathrm{~F}$-FDG PET tracers such as DOTATATE and HBED-CC initially developed for cancer detection are now also being used to determine the likely response to targeted radionuclide therapy using the same radiopharmaceutical labeled with a therapeutic radioisotope. Using multiparametric PET/MRI for such theranostic applications in place of PET/CT offers the potential for further improvements in the identification of patients most likely to benefit from subsequent targeted radionuclide therapy.

\section{Multiparametric PET/MRI for Precision Medicine in Place of Whole-Body MRI}

Whole-body MRI is now recommended for the assessment of asymptomatic myeloma patients and staging of solitary plasmacytomas (25), whereas whole-body MRI with diffusion imaging is emerging as a clinical tool for assessing therapeutic response in skeletal metastases (26). Although entailing additional radiation exposure, the use of PET/MRI for these indications in place of whole-body MRI could enable a wide range of established and novel PET tracers to be deployed for the assessment of tumor biology, with the possibility of providing an indication of likely treatment response as an adjunct to the diagnostic data afforded by the whole-body MRI examination. For example, amino acid PET has shown promise as a marker of disease activity in multiple myeloma (27) and if deployed using PET/MRI could potentially improve the identification of patients requiring treatment beyond whole-body MRI alone. However, the potential to append novel PET tracers to a diagnostic whole-body MRI is currently constrained by the fact that remarkably few PET radiotracers developed during the last decades have obtained regulatory approval for routine clinical use.

\section{HEALTH ECONOMIC IMPLICATIONS OF MULTIPARAMETRIC PET/MRI FOR PRECISION MEDICINE}

The use of multiparametric PET/MRI to identify subpopulations of patients with a greater or lesser likelihood of treatment failure or posttreatment recurrence offers the potential to optimize clinical care according to specific characteristics of an individual patient's tumor and so improve health outcomes and reduce health care expenditure. At present, there are insufficient data to undertake formal economic evaluations to identify precise circumstances under which multiparametric PET/MRI can replace PET/CT. However, modeling techniques such as decision tree analysis can be used to explore the outcomes that might be expected from deployment of multiparametric PET/MRI in a range of clinical scenarios. In this way, it is possible to determine the circumstances under which multiparametric PET/MRI has the potential to add sufficient value to replace PET/CT, given a set of plausible estimates for the model's input assumptions. These analyses can then act as a focus for future research concentrating on applications of multiparametric PET/MRI that offer the prospect of achieving clinical cost-effectiveness (28). The incremental cost of PET/MRI over PET/CT is a key determinant of likely costeffectiveness, and the impact of different cost estimates on likely cost-effectiveness can be evaluated using sensitivity analysis. For PET/MRI to have a realistic chance of achieving cost-effectiveness, this value needs to exceed current estimates for the incremental cost of PET/MRI. Allowing for the higher equipment cost, increased examination time, and expertise required, the incremental cost of PET/MRI is currently thought to be as high as $\$ 4,000$ per examination.

The identification of patients with a high likelihood of disease recurrence after treatment represents one example of precision medicine in which multiparametric PET/MRI can potentially add sufficient value to replace PET/CT. In this scenario, the results of PET/MRI could be used to deescalate surveillance in low-risk patients, thereby reducing health-care expenditure through fewer patients undergoing surveillance. Risk-adapted follow-up of patients after colorectal cancer surgery constitutes a specific implementation of this approach. Figure 1 summarizes a decision tree sensitivity analysis that explores this scenario. Strategies 1 and 2 use diagnostic PET/CT or PET/MRI to identify metastatic disease that would render patients unsuitable for surgery. The superior diagnostic performance for PET/MRI results in fewer patients identified as suitable for surgery. In strategy 3 , the addition of multiparametric PET/MRI enables identification of a low-risk subpopulation for whom surveillance can be avoided. The sensitivity analysis shows the most costeffective strategy for paired values for the probability of detecting early recurrence during surveillance ( $x$-axis) and the incremental cost of PET/MRI over PET/CT ( $y$-axis). Despite greater diagnostic performance than PET/CT, diagnostic PET/MRI alone is unlikely to be cost-effective under any circumstances unless the incremental cost over PET/CT is less than approximately $\$ 2,000$. However, multiparametric PET/MRI is potentially cost-effective over a wide range of probabilities for detecting recurrence, even with an incremental cost of $\$ 4,000$ (shaded zone). The analysis identifies the likelihood of detecting recurrence in low-risk in comparison to high-risk patients as a key unknown that requires evaluation by future research. The estimate of 1:3 used in this analysis is comparable to that reported in a prospective evaluation of risk-adapted follow-up of colorectal cancer in which pathologic features were used to stratify patients (29).

The greatest promise for economic benefit from the use of multiparametric PET/MRI in precision medicine lies in identifying patients unlikely to receive sufficient benefit from a particular treatment. Selection of patients for immune checkpoint blockade constitutes a specific implementation of this approach for which histologic techniques are currently under evaluation (30). A similar concept could be applied to theranostic applications of PET/MRI, with multiparametric imaging further refining the selection of patients for radionuclide 


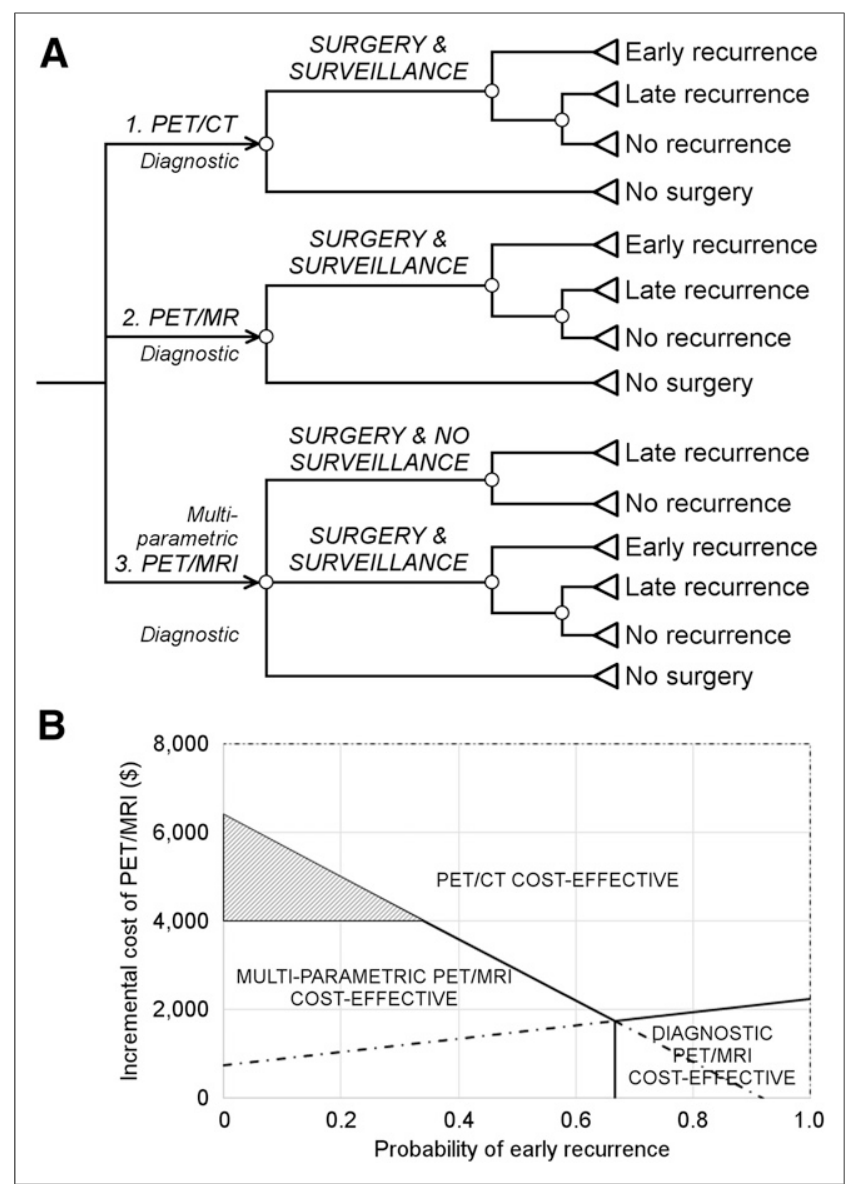

FIGURE 1. Illustrative decision tree (A) and sensitivity analysis (B) exploring health economic conditions under which multiparametric PET/MRI could add value by enabling risk-adapted postsurgical surveillance for early detection and treatment of recurrence. (Assumptions: likelihood of detecting metastatic disease, 20\% for PET/CT and 25\% for PET/MRI; likelihood of detecting recurrence in low-risk patients, one third of that for high-risk patients; incremental costs per patient for surveillance and treatment of early recurrence, $\$ 15,000$ and $\$ 30,000$, respectively; incremental benefit per patient from early treatment of recurrence, 1.5 qualityadjusted life year; health purchaser's willingness to pay, $\$ 50,000$ per quality-adjusted life year. Solid lines indicate where 2 strategies have equal net monetary benefit.)

therapy. Figure 2 summarizes a decision tree sensitivity analysis that explores such scenarios. In strategies 1 and 2, diagnostic PET/CT is used to identify patients unsuitable for chemotherapy but cannot determine the likelihood of response to targeted therapy. Therefore, all patients must receive either standard therapy (chemotherapy A—strategy 1) or targeted therapy (chemotherapy B-strategy 2). In strategy 3, multiparametric PET/MRI has the same diagnostic capability as PET/CT but adds value by identifying a subpopulation of patients most likely to respond to targeted therapy. The sensitivity analysis shows the most cost-effective strategy for paired values for prevalence of responders ( $x$-axis) and the incremental cost per patient of PET/MRI over PET/CT ( $y$-axis). Multiparametric PET/MRI is potentially cost-effective over a wide range of values for prevalence of responders, even with an incremental cost of $\$ 4,000$ (shaded zone). There are no conditions under which PET/MRI would be cost-effective if used for diagnosis alone.

\section{CHALLENGES FACING THE USE OF PET/MRI FOR PRECISION MEDICINE IN ONCOLOGY}

The success of the above approach is predicated on meeting several challenges that face the use of multiparametric imaging within the precision medicine paradigm in oncology. Technologic issues include the lack of purpose-designed computer software for extraction and reporting of multiparametric imaging data, and methods for identifying and validating multiparametric imaging signatures are currently underdeveloped. It is also essential to establish protocols that ensure measurement consistency of multiparametric PET/MRI across different imaging platforms. There is a requirement for training of radiologists and technical staff

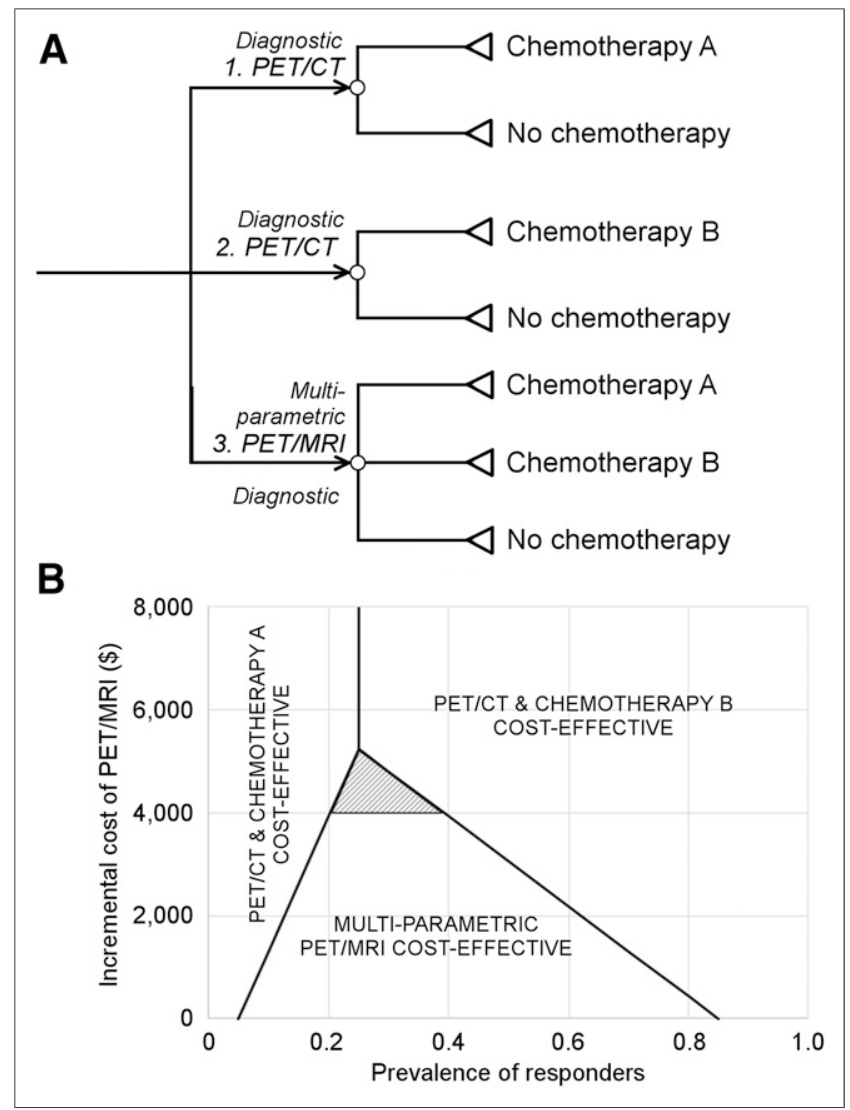

FIGURE 2. Illustrative decision tree (A) and sensitivity analysis (B) to identify health economic conditions under which multiparametric $\mathrm{PET} / \mathrm{MRI}$ could add value by identifying a subpopulation enriched for response to targeted therapy. (Assumptions: likelihood of receiving chemotherapy, 70\%; false-positive and false-negative rates for $\mathrm{PET} / \mathrm{MRI}$ identification of responders, $15 \%$ and $5 \%$, respectively; incremental cost and benefit per patient for chemotherapy $B$ over chemotherapy A, $\$ 12,500$ and 1.0 quality-adjusted life year, respectively; health purchaser's willingness to pay, $\$ 50,000$ per quality-adjusted life year. Solid lines indicate where 2 strategies have equal net monetary benefit.) 
who may be unfamiliar with the concepts of precision medicine. Because the use of imaging for precision medicine represents a novel paradigm within cancer care, an initial reluctance by clinicians to accept this role for imaging can be also anticipated. It will also be necessary to demonstrate the value of multiparametric imaging in comparison to competing technologies such as tissue and blood-based assays.

\section{CONCLUSION}

PET/MRI is a remarkable hybrid imaging device that combines the advantages of 2 powerful modalities. However, the diagnostic benefits of this combination appear to be limited, and supplementary clinical roles must be established for PET/MRI to become a routine modality within clinical cancer care. Although the challenges involved should not be underestimated, the use of multiparametric PET/MRI for precision medicine not only can increase the clinical value accruing from deployment of this technology but also can potentially establish it as a cost-effective modality within clinical cancer care.

\section{DISCLOSURE}

Kenneth Miles is a shareholder in Feedback PLC which supplies software for CT texture analysis. No other potential conflict of interest relevant to this article was reported.

\section{REFERENCES}

1. Spick C, Herrmann K, Czernin J. ${ }^{18}$ F-FDG PET/CT and PET/MRI perform equally well in cancer: evidence from studies on more than 2,300 patients. J Nucl Med. 2016;57:420-430.

2. Kuwert T, Ritt P. PET/MRI and PET/CT: is there room for both at the top of the food chain? Eur J Nucl Med Mol Imaging. 2016;43:209-211.

3. Krishnaraj A, Weinreb JC, Ellenbogen PH, Allen B, Norbash A, Kazerooni EA. The future of imaging biomarkers in radiologic practice: proceedings of the thirteenth annual ACR forum. J Am Coll Radiol. 2014;11:20-23.

4. Sala E, Micco M, Burger IA, et al. Complementary prognostic value of pelvic magnetic resonance imaging and whole-body fluorodeoxyglucose positron emission tomography/computed tomography in the pretreatment assessment of patients with cervical cancer. Int J Gynecol Cancer. 2015;25:1461-1467.

5. Schillaci O, Urbano N. Personalized medicine: a new option for nuclear medicine and molecular imaging in the third millennium. Eur J Nucl Med Mol Imaging. 2017;44:563-566

6. Chowdhury R, Ganeshan B, Irshad S, et al. The use of molecular imaging combined with genomic techniques to understand the heterogeneity in cancer metastasis. Br J Radiol. 2014;87:20140065.

7. Strauss LG, Koczan D, Klippel S, et al. Impact of angiogenesis-related gene expression on the tracer kinetics of ${ }^{18} \mathrm{~F}-\mathrm{FDG}$ in colorectal tumors. $\mathrm{J} \mathrm{Nucl} \mathrm{Med}$. 2008;49:1238-1244.

8. Kawada K, Toda K, Nakamoto Y, et al. Relationship between ${ }^{18} \mathrm{~F}-\mathrm{FDG}$ PET/CT scans and KRAS mutations in metastatic colorectal cancer. J Nucl Med. 2015; 56:1322-1327.
9. Strauss LG, Dimitrakopoulou-Strauss A, Koczan D, Pan L, Hohenberger P. Correlation of dynamic PET and gene array data in patients with gastrointestinal stromal tumors. ScientificWorldJournal. 2012;2012:721313.

10. Joo YH, Yoo IR, Cho KJ, Park JO, Nam IC, Kim MS. Preoperative ${ }^{18}$ F-FDG $\mathrm{PET} / \mathrm{CT}$ and high-risk HPV in patients with oropharyngeal squamous cell carcinoma. Head Neck. 2014;36:323-327.

11. Kendi AT, Magliocca K, Corey A, et al. Do ${ }^{18}$ F-FDG PET/CT parameters in oropharyngeal and oral cavity squamous cell carcinomas indicate HPV status? Clin Nucl Med. 2015;40:e196.

12. Higashi K, Ueda Y, Ikeda R, et al. P-glycoprotein expression is associated with FDG uptake and cell differentiation in patients with untreated lung cancer. $\mathrm{Nucl}$ Med Commun. 2004;25:19-27.

13. Seo S, Hatano E, Higashi T, et al. Fluorine-18 fluorodeoxyglucose positron emission tomography predicts tumor differentiation, P-glycoprotein expression, and outcome after resection in hepatocellular carcinoma. Clin Cancer Res. 2007;13:427-433.

14. Mazurowski MA, Zhang J, Grimm LJ, et al. Radiogenomic analysis of breast cancer: luminal B molecular subtype is associated with enhancement dynamics at MR imaging. Radiology. 2014;273:365-372.

15. Agner SC, Rosen MA, Englander S, et al. Computerized image analysis for identifying triple-negative breast cancers and differentiating them from other molecular subtypes of breast cancer on dynamic contrast-enhanced MR images: a feasibility study. Radiology. 2014;272:91-99.

16. Youk JH, Son EJ, Chung J, Kim JA, Kim EK. Triple-negative invasive breast cancer on dynamic contrast-enhanced and diffusion-weighted MR imaging: comparison with other breast cancer subtypes. Eur Radiol. 2012;22:1724-1734.

17. Thoeny HC, Ross BD. Predicting and monitoring cancer treatment response with diffusion-weighted MRI. J Magn Reson Imaging. 2010;32:2-16.

18. Dang M, Lysack JT, Matthews TW, et al. MRI texture analysis predicts p53 status in head and neck squamous cell carcinoma. AJNR Am J Neuroradiol. 2015;36:166-170

19. Michoux N, Van Den Broeck S, Lacoste L, et al. Texture analysis on MR images helps predicting non-response to NAC in breast cancer. BMC Cancer. 2015;15:574.

20. Antunes J, Viswanath S, Rusu M, et al. Radiomics analysis on FLT-PET/MRI for characterization of early treatment response in renal cell carcinoma: a proof-ofconcept study. Transl Oncol. 2016;9:155-162.

21. Mankoff DA, Dunnwald LK, Gralow JR, et al. Blood flow and metabolism in locally advanced breast cancer: relationship to response to therapy. $\mathrm{J} \mathrm{Nucl} \mathrm{Med}$. 2002;43:500-509.

22. Dimitrakopoulou-Strauss A, Strauss LG, Burger C, et al. Prognostic aspects of ${ }^{18}$ F-FDG PET kinetics in patients with metastatic colorectal carcinoma receiving FOLFOX chemotherapy. J Nucl Med. 2004;45:1480-1487.

23. Yang Z, Zan Y, Zheng X, et al. Dynamic FDG-PET imaging to differentiate malignancies from inflammation in subcutaneous and in situ mouse model for non-small cell lung carcinoma (NSCLC). PLoS One. 2015;10:e139089.

24. Tsaknakis B, Schaefer IM, Schwörer H, et al. Long-lasting complete response of metastatic melanoma to ipilimumab with analysis of the resident immune cells. Med Oncol. 2014;31:813.

25. Rajkumar SV, Dimopoulos MA, Palumbo A, et al. International Myeloma Working Group updated criteria for the diagnosis of multiple myeloma. Lancet Oncol. 2014;15:e538-e548.

26. Lecouvet FE, Larbi A, Pasoglou V, et al. MRI for response assessment in metastatic bone disease. Eur Radiol. 2013;23:1986-1997.

27. Lapa C, Knop S, Schreder M, et al. ${ }^{11} \mathrm{C}$-methionine-PET in multiple myeloma: correlation with clinical parameters and bone marrow involvement. Theranostics. 2016;6:254-261.

28. Chilcott J, Brennan A, Booth A, Karnon J, Tappenden P. The role of modelling in prioritising and planning clinical trials. Health Technol Assess. 2003;7:iii, 1-125.

29. Secco GB, Fardelli R, Gianquinto D, et al. Efficacy and cost of risk-adapted follow-up in patients after colorectal cancer surgery: prospective, randomized controlled trial. Eur J Surg Oncol. 2002;28:418-423.

30. Aguiar PN, Perry L, Penny-Dimr J, et al. The effect of PD-L1 testing on the costeffectiveness and economic impact of immune checkpoint inhibitors for the second-line treatment of NSCLC. Ann Oncol. 2017;28:2256-2263. 